\title{
The Scottish Electricity Dispatch Model
}

\author{
Shona Pennock, Simon Gill, Keith Bell \\ University of Strathclyde \\ Glasgow, UK \\ shona.pennock@strath.ac.uk,simon.gill@strath.ac.uk, keith.bell@strath.ac.uk
}

\begin{abstract}
This paper presents the Scottish Electricity Dispatch Model (SEDM), a cost minimization power system optimization model designed as a tool to investigate potential development trajectories of the Great Britain (GB) power system with particular focus on the Scottish region. Results of SEDM studies investigating the impact of low carbon policies on carbon emissions, generation portfolios and system costs are described. Two potential policies are investigated: carbon pricing and emissions limits, compared to a base scenario with neither of these policies included, but featuring financial support for low carbon generation in line with current GB policy. Model results suggest that carbon emissions could be reduced by up to $95.6 \%$ by 2050 with strict emissions limits, achieved largely through an eighteen fold increase in renewable generation capacity. This results in an increase in system costs (including carbon pricing costs) of up to $23.4 \%$ compared to the base case, in which carbon emissions increase by $\mathbf{1 3 0 . 8 \%}$ from present day to 2050 . This suggests current policies will be insufficient to achieve a reduction in carbon emissions to the level suggested by the UK's Committee on Climate Change (CCC).
\end{abstract}

Index Terms-power system modelling, investment planning, generation planning, dispatch modelling, carbon emissions targets

\section{INTRODUCTION}

Lowering carbon emissions is a key target of many European states, due to the approaching deadline for the 2020 targets [1]. In Great Britain (GB), political targets for lowering emissions at least cost while safeguarding security of supply resulted in Electricity Market Reform (EMR). EMR introduced new subsidies for low carbon generation in the form of a feed-in tariff with contracts for difference (CfDs) and a capacity market designed to ensure sufficient dispatchable generation [2]. The transition to EMR involves a competitive auction for subsidies in which low carbon projects bid for available CfDs, limited by the funds available under the Levy Control Framework [3]. Other factors impacting on the investment in generation in GB include the current market conditions and ease of access to market. Much of the wholesale power market trading in GB occurs as long term bilateral trading, resulting in fairly low market liquidity. Data from Ofgem, the regulator for gas and electricity markets in $\mathrm{GB}$, shows that $83 \%$ of electricity trading volumes were from bilateral trades in 2015 [4]. Ofgem has found the GB wholesale market to be comparably competitive with other European markets however [5]. Ofgem has also stated that recent examples can be found of both supply and generation being able to enter and exit the market [5].

In addition to market influences within GB, the development of a European single energy market (ESEM) could also impact on investment in generation. The key concept behind the ESEM is to improve liquidity in crossborder trading, by use of market coupling and the implicit auction of network capacity [6]. As a mainly bilateral market, GB could be impacted by the focus on power exchange trading in the ESEM network codes. In addition, congestion management measures such as bidding zones could impact on generator revenues, depending on the zonal configuration [7].

The Scottish Electricity Dispatch Model (SEDM) has been developed as a least-cost power system optimization model with the aim of increased resolution over issues related to the Scottish section of the GB power system compared with previous GB models used by policy makers [8],[9]. This paper will outline SEDM base case inputs and capabilities, before going on to show results from example scenarios investigating the impact of carbon reduction policies on system costs, investment in generation and carbon emissions. A discussion of the limitations of the model's capabilities is also included. It should be noted that Northern Ireland, although politically part of the UK, is electrically part of the single energy market on the island of Ireland so this paper is deliberately referring to the GB power system rather than the UK.

\section{The SCOTTISH ElECTRICITY DISPATCH MODEL}

\section{A. Model Summary}

The SEDM model is based on ORDENA plus, an existing generic electricity market dispatch and investment tool developed by AF-Mercados [10], populated with detailed information to represent the Scotland and GB power system for use by the Scottish Government [11]. The model comprises of two modules - long term planning and short term unit commitment. This paper focuses on the long term planning module.

SEDM simulates long-term investment decisions in generation and inter-area transmission capacity based on costminimization. The objective function minimizes the net present value (NPV) of system costs over a planning horizon of present day until 2050. Costs include: capital and operational costs for generation and transmission, carbon costs and financial support granted to low carbon generation. This objective function is represented in (1):

$$
\min \sum_{b, y}(\mathrm{Cap}+\mathrm{Op}+\mathrm{Em}-\mathrm{Subs})
$$

The summation is over all time periods (within-year blocks $b$ and years $y$ ) and all costs are in NPV in 2014 prices. The costs are broken down as follows:

Cap are all capital costs incurred in a particular block and year for generation, transmission and demand (if demand-side management is considered) 
- $\quad O p$ are all operational costs across all blocks

- $\quad$ Em are carbon emissions costs

- $\quad$ Subs are subsidies paid to generators such as CfDs and renewable obligation certificates (ROCs) [12], entered into the model as negative costs.

Various political targets, government subsidies and technical constraints, discussed below, can be enabled to allow a range of scenarios to be simulated.

The model comprises fourteen nodes representing GB, ten of which are used to represent areas in Scotland. The model nodes are illustrated in figure 1. Each node has associated generation, demand and transmission capacity to other nodes based on present day GB system capacities, as well as a set of options for future development [13].

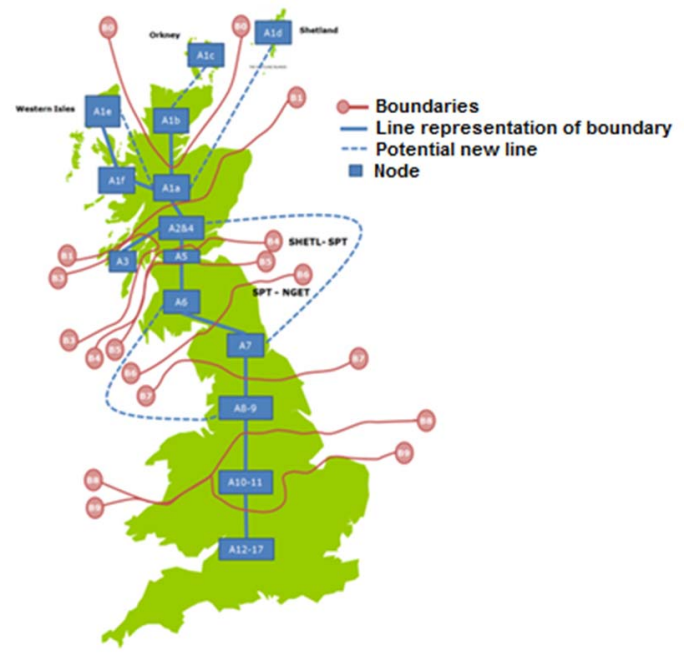

Figure 1. Illustration of SEDM nodes, from [11]

\section{B. Modelling of generation and demand}

Anticipated retirement dates of existing generation are input, in addition to earliest generation entry dates of publicly announced projects. Additional investment in generation is selected from a portfolio of candidate projects. To differentiate the risk associated with development of different technologies, technology-specific discount rates are used in addition to the overall discount rate [14].

Demand and renewable generation profiles are simplified into blocks in SEDM, as a chronological representation of demand and generation until 2050 would prove exceedingly computationally intensive. Four load blocks are used per year, one short peak block representing absolute peak demand and three longer blocks representing average mid-range and baseload demand. Figure 2 illustrates the aggregated load duration blocks used.

Wind generation is represented by use of wind capacity factor blocks within load blocks: each load block is split into three smaller blocks, representing high, medium and low capacity factors at each demand level, as shown in figure 2. Wind speed data from a mesoscale model is used to create the capacity factors for each of the twelve wind blocks for each node [15].

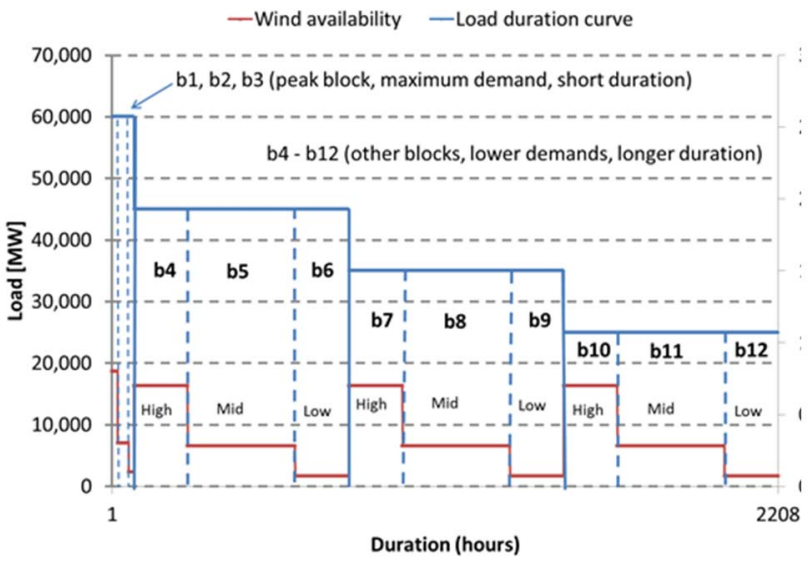

Figure 2. Comparison of load duration blocks and wind availability blocks, from [11]

Generation from pumped hydro storage is also represented by comparison of generation capability and load blocks. It is assumed that pumping will only occur during the baseload load blocks and that most of the generation will take place during the peak block.

Thermal generation in the long term model is represented by linear operating costs based on a gross operational efficiency. Both planned and forced outages of generation are modelled by annual availability factors [14].

\section{Constraints and modelling of the network}

The model can be run with transmission inter-area transfer limits between nodes enabled or disabled. When transmission constraints are enabled, transmission is modelled in a similar manner to generation, with current transfer capacity at model boundaries input, planned upgrades included with a predicted entry year and various additional candidate projects available to be built if required by the system. Investment in candidate transmission upgrades is optimized in parallel with investment in candidate generation projects. Transmission Network Use of System (TNUoS) charges are added in to the objective function for unconstrained runs, used as a proxy for transmission capital costs in scenarios where the network is not modelled explicitly. Transmission losses are modelled in all scenarios as additional demand.

Kirchhoff's first law is input as a model constraint, i.e. that all power entering the system at a geographical location from generation at that location or via the network must equal that leaving as demand or exports. However, Kirchhoff's voltage law is neglected.

Interconnectors to GB are modelled by fixed time series of transfers derived from historical data or dynamic dispatch via a combination of linked 'pseudo' generation and demand attributed to the appropriate node. Interconnector capacity is limited by the combination of demand and availability at the pseudo generator. The interconnector with France, for example, is represented as a blocked load duration curve, an off-peak pseudo nuclear generator and a peaking pseudo gas plant. This is intended to allow the relative prices of generation in the interconnected areas to determine dispatches and hence interconnector flows. 
Measures to maintain generation adequacy are modelled by use of a de-rated capacity margin. Generation is allocated a de-rated capacity to represent peak availability [16], and a constraint can be activated to ensure that a user-input annual peak de-rated capacity margin must be met. Capacity markets can also be represented in SEDM as capacity payments to particular sets of generators.

Annual development limits, such as maximum investment (in currency) and build rates (maximum installed capacity) are included as constraints in the model inputs. Annual operational limits can also be included, for example maximum fuel consumption and minimum/maximum power generation per year. These limits are intended to represent restrictions due to the supply chain and planning procedures, so that a representative technology mix is reached instead of the model only choosing to invest in the cheapest type of generation and building unrealistic amounts of a single technology.

\section{Model Outputs}

Model outputs include an investment schedule in generation and transmission, total costs for investment, operation and subsidies, dispatch by load/wind block, interarea power flows, carbon emissions and the annual system marginal prices, represented by a demand-weighted average of the nodal marginal prices. Due to the assumptions inevitably involved with developing a model of this complexity and uncertainties associated with using predictions of demand and fuel costs until 2050, analysis by scenario comparison is by far the most credible way to evaluate results.

\section{MODELLED SCENARIOS}

\section{A. Model Basecase}

A 2015 base case scenario for GB has been established by the authors. This includes a representation of current, planned and candidate generation and transmission capacity in the GB system as of August 2015 and associated capital and operational costs, along with trajectories for future fuel prices, load growth and emissions prices [17]. The 2015 base case represents a view of these inputs based on several reports from sources such as National Grid and the UK Government Department of Energy and Climate Change (DECC) [13] [19].

\section{B. Carbon Targets Scenarios}

A number of policy tools have been used by various governments in order to encourage the decarbonisation of the power system. These include direct, legally enforceable limits on emissions, emissions taxes and subsidy support for low carbon generation, SEDM has the ability to model each of these policy interventions concurrently or separately.

In order to study the potential effectiveness of each of these policies on decarbonisation and ability to meet low carbon targets, three scenarios were run:

1. A base case with no policy intervention except the existing low carbon subsidy regime. This can be represented as an objective function with no additional costs or constraints due to emissions, and subsidies modelled as negative costs as shown in (2):

$$
\min \sum_{b, y}(\mathrm{Cap}+\mathrm{Op}-\mathrm{Subs})
$$

2. A carbon pricing scenario in which existing subsidies for low carbon generators are complemented with carbon pricing figures taken from the carbon price floor set out in the UK budget at $£ 70 / \mathrm{tCO}_{2}$ in 2030 [20], increasing linearly from the $£ 18 / \mathrm{tCO}_{2}$ cap until 2020 [21]. This can be represented with an objective function including additional operational costs due to carbon pricing, as shown in (1).

3. Carbon pricing and low carbon subsidies combined with strict emissions limits from 2030 onwards based on input from the committee on climate change (CCC) carbon budgets for $\mathrm{GB}$, in which emissions from power stations are $16 \mathrm{MtCO}_{2}$ by 2030 , with a linear reduction to $5 \mathrm{MtCO}_{2}$ by 2050 [22]. This can be represented with an objective function including emissions costs due to carbon pricing (used to limit emissions until strict emissions limits occur in 2030) and a constraint limiting the annual emissions produced, as shown in (3):

$$
\begin{gathered}
\min \sum_{b, y}(\mathrm{Cap}+\mathrm{Op}+\mathrm{Em}-\mathrm{Subs}) \\
\operatorname{Vol}_{E m} \leq E m_{\text {targ }}
\end{gathered}
$$

where $V l_{E m}$ is the annual volume of emissions produced for each year modelled and $E m_{\text {targ }}$ is the annual emissions targets input to the model for each corresponding year.

All scenarios have the transmission network constraint enabled between nodes and use historical data to represent interconnectors.

\section{RESUlts}

Three sets of outputs for the scenarios modelled are reported here: total installed generation capacity, installed renewable generation capacity and $\mathrm{CO}_{2}$ emissions. Installed capacity in 2015 represents the current generation fleet in GB, whilst future years include the model investment decisions for new generation capacity in subsequent years and the retirement of existing plant.

Figure 3 illustrates the total installed renewable capacity for each of the three scenarios described in section III-C. The base case results show continued growth in renewables until 2020 due to wind projects already in development and planning, and then effectively no further investment in renewables after 2020. The carbon pricing scenario shows a moderate increase in renewable installed capacity until the early 2020s, and then little new capacity development until the late 2020s. There is a sharp peak in development at 2030 due to a higher carbon price coming into effect in that year, and then a steady decline in renewables capacity until 2050. The carbon pricing scenario has $37.9 \%$ greater installed renewable capacity than the base case scenario by 2030 and $425.7 \%$ by 2050. Other low carbon generation (nuclear and carbon capture and storage technologies) also increase in installed capacity, by $178.9 \%$ in 2050 compared with the base case. The emissions limits scenario shows a similar trajectory to the carbon pricing scenario until 2030. This is due to the fact that both scenarios include carbon pricing across this period, whilst emissions limits are only binding after 2030. For 2028 to 2032 the emissions limits scenario sees a huge expansion of renewable generation with installed capacity rising by approximately $23 \mathrm{GW}$ over four years, as the model invests to 
meet the emissions targets in 2030 and beyond. Renewable capacity then declines until 2045, before climbing again in until 2050. The emissions limits scenario has $54.5 \%$ greater installed capacity of renewables by 2030 when compared with the base case, and this figure is $1812.0 \%$ by $2050-81.7 \mathrm{GW}$ compared with $4.3 \mathrm{GW}$. This scenario has an increase of $234.3 \%$ of installed capacity of other low carbon technologies by 2050 compared with the base case. The difference in installed capacity between these three scenarios is due to the fact that without any financial incentives or constraints for low carbon technologies, the model will invest in the generation with the cheapest levelised costs (coal and gas) rather than renewable generation with higher levelised costs. Carbon pricing results in an increase in installed renewable capacity when compared to the base case, but the emissions limits constraint causes a much larger investment in renewable capacity. The carbon pricing and emissions limits scenarios are similar until 2030 because prior to this the level of emissions limits are above the level achieved via the use of carbon pricing and the residual impact of existing renewable generation.

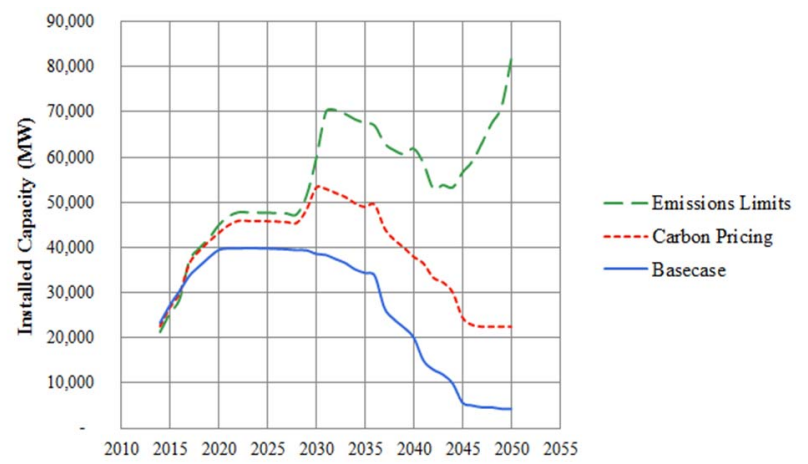

Figure 3. Installed capacity from renewable generation for the base case, carbon pricing and emissions limits scenarios

Whilst Figure 3 shows the capacity of all renewable generation, it is interesting to identify the component of this provided by wind. Figure 4 illustrates the installed capacity from wind generation in each of the scenarios. In this case the emissions limits and carbon pricing scenarios produce similar results until 2040, at which point there is $255 \%$ more installed wind capacity when compared with the base case scenario. When compared with Figure 3 it is clear that a major component of the spike in renewable capacity in the emissions limits scenario at 2030 is due to technologies other than wind. The model contains build limits for each technology to represent supply chain constraints. As such, the model must invest in a varied mix of low carbon generation to meet the stringent emissions limits, including solar, biomass and marine technologies such as wave and tidal. In this particular case all of the Round 1 and 2 offshore wind projects input to the model as candidate generation are built to maximum capacity, as well as the Scottish Round 3 offshore wind projects. At this point the model builds solar power in the south of England rather than investing in the more southerly Round 3 offshore wind sites, as the lower capacity factors compared with the Scottish wind resource result in an increased levelised cost.

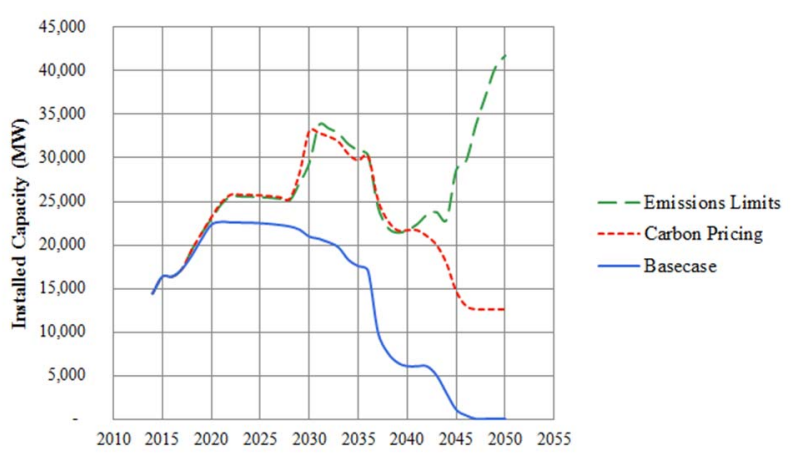

Figure 4. Installed capacity from wind generation for the base case, carbon pricing and emissions limits scenarios

Figure 5 shows the annual $\mathrm{CO}_{2}$ emissions for each of the three scenarios. It can be seen that the base case scenario emissions increase steadily from 2020 , once the renewables projects currently in planning have been completed. In the carbon pricing scenario annual emissions decline until 2023 and then remain fairly steady. In the emissions limits scenario annual emissions decline until 2030 before a shallower decline until 2050. Carbon emissions are found to reduce by up to $95.6 \%$ by 2050 when comparing the emissions limits scenario with the base case.

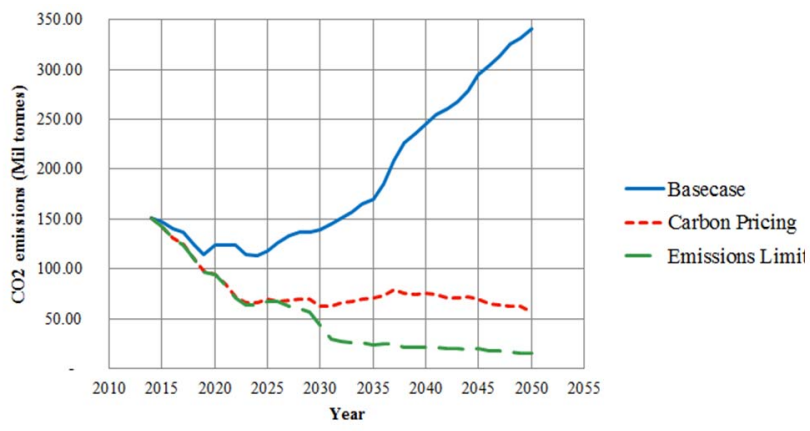

Figure 5. Annual carbon emissions for the base case, carbon pricing and emissions limits scenarios

System costs increase by $23.4 \%$ between the emissions limits and base case scenarios by 2050 . This is due to the additional model constraints resulting in the investment of more expensive generation technologies, and there being no cost associated with carbon emissions in the base case scenario.

Overall, the results suggest that long term carbon reduction will not occur in a base case simulation representing the current GB system and including current subsidies to low carbon technologies. Simulations representing policies such as carbon pricing or the legal enforcement of emissions limits did result in the reduction of carbons emissions, through increased investment in renewable technologies.

\section{Discussion}

There are various challenges involved with the interpretation of results from such a large, complex model. As this paper has shown, model results can change greatly depending on the input scenario. The results produced are highly sensitive to emissions constraints and other inputs, such as demand forecast, fuel prices and available spend for 
subsidies. The approach used in SEDM of minimum cost modelling of future scenarios in a liberalized market such as that in GB does not necessarily take account of the range of factors that influence actual investment. These include market power, bilateral trading agreements, government targets, policy instruments and planning procedures. Results must be understood with these limitations in mind.

Technology-specific constraints on annual investment and generation are used in SEDM to represent the capabilities of the supply chain, available resources and planning procedures, but it is impossible to be totally accurate about the future level of these constraints. In the emissions limits scenario results the maximum annual installed capacity (MW) is a binding constraint from 2022 until 2033, this is observed as a large peak in installed capacity in figures 3 and 4 . This amount of investment in wind and solar technologies, especially between 2028-2032, would likely be difficult to achieve due to supply chain and investment constraints. Reducing the value of the technology-specific annual build constraints would result in the model having to invest in low carbon technologies to meet emissions constraints over a longer time period. Further work with the model could include an assessment of such constraints.

There are also some general issues with large scale optimal modelling of power systems which the use of SEDM reveals. These include the issue of meeting computation requirements, such as reasonable simulation times. For example, enabling transmission constraints results in a more realistic dispatch, but also takes a great deal more time to run scenarios than the unconstrained model. A model run with transmission constraints and transmission upgrades enabled takes approximately three hours, whereas with an unconstrained network a single simulation takes $10-15$ minutes. The reason for this is that modelling the transmission network requires the introduction of additional decision variables, namely the capacity of transmission connection build in any one year. When modelling large complex systems there is a trade-off involved with simplifying the simulation problem to reduce the number of decision variables. Candidate generators are treated as continuous variables in the model for this reason.

\section{CONCLUSIONS}

As has been demonstrated in this paper, the SEDM tool can be used to compare the impact of different carbon reduction scenarios. Scenario comparison allows the user to compare numerous low carbon policies to a base case, changing one input or constraint per scenario to judge the potential impact of each carbon reduction policy on the GB system. The results indicate that current policies will be insufficient to achieve a reduction in carbon emissions to the level suggested by the CCC, even with the addition of the current carbon price floor. Various model limitations and assumptions have also been discussed, which must be considered when interpreting these results.

\section{ACKNOWLEDGMENTS}

The authors gratefully acknowledge the Scottish Government for allowing access to the SEDM model. The Scottish Government has not validated the results, nor do they specifically represent government policy. The first author also acknowledges financial support from the Engineering and Physical Sciences Research Council through the Centre for Doctoral Training in Wind Energy Systems (grant EP/L016680/1). The second author acknowledges support from ClimateXChange http://www.climatexchange.org.uk/

\section{REFERENCES}

[1] European Commission, “2020 climate \& energy package,” 2009. Available:

http://ec.europa.eu/clima/policies/strategies/2020/index_en.htm.

[2] Department of Energy and Climate Change, "Planning our electric future: a White Paper for secure, affordable and low-carbon electricity," July 2011. Available:

https:/www.gov.uk/government/publications/planning-our-electricfuture-a-white-paper-for-secure-affordable-and-low-carbon-energy

[3] National Audit Office, "The Levy Control Framework," 27 Nov 2013. Available: https://www.nao.org.uk/press-releases/levy-controlframework/

[4] Ofgem, "Monitoring the market: Wholesale market indicators," 2015. Available: https://www.ofgem.gov.uk/monitoring-market/wholesalemarket-indicators

[5] Ofgem, "Wholesale Energy Markets in 2015," 9 September 2015. Available: https://www.ofgem.gov.uk/publications-and-updates/ wholesale-energy-markets-2015

[6] ACER, "Framework Guidelines on Capacity Allocation and Congestion Management for Electricity,” 29 July 2011.

[7] Energy UK. "European electricity network codes - Factsheet," January 2015. Available: https:/www.energy-uk.org.uk/publication.html?task= file.download\&id=5072

[8] Department of Energy and Climate Change, "DECC Dynamic Dispatch Model,” May 2012. Available: https://www.gov.uk/ government/publications/dynamic-dispatch-model-ddm

[9] The Scottish Government, "Electricity Generation Policy Statement," 2013. Available: http://www.gov.scot/Topics/Business-Industry/ Energy/EGPSMain

[10] AF-Mercados, "Powerful Targets: Exploring the relative cost of meeting decarbonisation and renewables targets in the British power sector," 2012. Available: http://www.templar.co.uk/downloads/ Powerful_Targets.pdf

[11] AF Mercados EMI, "Scottish Electricity Dispatch Model User Guide: Model Design and Data Sourcing," Unpublished, 2013.

[12] Ofgem, "Renewables Obligation (RO)," 2015. Available: https://www.ofgem.gov.uk/environmental-programmes/renewablesobligation-ro

[13] National Grid, "Electricity Ten Year Statement," 2014. Available: http://www2.nationalgrid.com/uk/industry-information/future-ofenergy/

[14] Department of Energy and Climate Change, "Electricity Generation Costs," December 2013. Available: https://www.gov.uk/government/ publications/decc-electricity-generation-costs-2013

[15] S. Hawkins, "A High Resolution Reanalysis of Wind Speeds over the British Isles for Wind Energy Integration," Ph.D. thesis, Univ. Edinburgh, 2012.

[16] Ofgem, "Electricity Capacity Assessment 2014," 30 June 2014

[17] Department of Energy and Climate Change, "Updated energy and emissions projections: 2014" 25 September 2014.

[18] Department of Energy and Climate Change, "Renewable Energy Planning Database: August 2015," August 2015. Available: https://www.gov.uk/government/publications/renewable-energyplanning-database-monthly-extract.

[19] National Grid, "DemandData_Historic-2014," 14 July 2015. Available: http://www2.nationalgrid.com/UK/Industry-information/Electricitytransmission-operational-data/Data-Explorer/

[20] HM Treasury, "Carbon price floor consultation: the Government response," March 2011.

[21] HM Revenue and Customs, "Carbon price floor: reform and other technical amendments," 19 March 2014.

[22] Committee on Climate Change, "The Fourth Carbon Budget - reducing emissions through the 2020s," December 2010. 